\title{
Do mothers understand healthy eating principles for low-birth-weight infants?
}

\author{
Jane B Morgan ${ }^{1, *}$, Peter Williams ${ }^{2}$, Keith D Foote ${ }^{3}$ and Lynne D Marriott ${ }^{1}{ }^{1}$ \\ ${ }^{1}$ School of Biomedical and Molecular Sciences, University of Surrey, Guildford, Surrey, GU2 7XH, UK: ${ }^{2}$ Department \\ of Mathematics and Statistics, University of Surrey, Guildford, Surrey, GU2 7XH, UK: ${ }^{3}$ Department Paediatrics and \\ Child Health, Royal Hampshire County Hospital, Winchester, Hampshire, SO22 5DG, UK
}

Submitted 11 July 2005: Accepted 26 0ctober 2005

\begin{abstract}
Objectives: To describe feeding patterns and mothers' perceptions of desirable feeding practices in low-birth-weight (LBW) infants after hospital discharge in England and to test for the association of inappropriate practices/perceptions with identifiable demographics.

Design: Postal questionnaires from 198 mothers of LBW infants in London, Liverpool and Winchester were analysed regarding infant demographics, mothers' demographics, infant milks used, solid feeding practices and mothers' perceptions of infant feeding practices.

Results: At birth, the median weight, independent of gestational age, was $1.80 \mathrm{~kg}$ (range $0.6-2.50 \mathrm{~kg}$ ) and $37.1 \%$ of infants received breast milk exclusively; $83.7 \%$ were breast-fed at some stage, a practice favoured more by first-time mothers $(90.2 \%)$ than multiparous mothers $(73.4 \%)(P=0.004)$ as well as by mothers of higher social groups $(P=0.019)$. The median age of solid food introduction was 17 postnatal weeks (range 8-36 weeks); the timing correlated strongly with infant birth weight $(P<0.001)$. A high-fibre diet and a low-fat diet were incorrectly considered important for their infants by $67.1 \%$ and $51.6 \%$ of mothers, respectively. Regarding a high-fibre diet as important was associated with being a multiparous mother $(P=0.006)$, while regarding a low-fat diet as important was associated with low social group $(P=0.018)$. A quarter of mothers did not consider 'plenty of calories' to be important for their infants, reflecting similar attitudes in 1990 for mothers of term infants; this incorrect attitude exhibited an association with being a first-time mother $(P=0.047)$.

Conclusions: Infant feeding practices were very variable, showing poor concordance with national and international recommendations for term infants. In selecting foods for their infants, many mothers appeared to be applying principles more appropriate to planning a healthy diet for adults. There is an urgent need to develop and implement evidence-based guidelines for feeding LBW infants after hospital discharge, with special attention given to multiparous mothers as well as mothers from lower social groups.
\end{abstract}

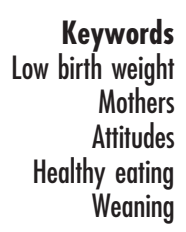

There is a growing recognition that diet in infancy has an important effect on later health ${ }^{1}$. This is especially true of low-birth-weight (LBW) infants, for whom early nutritional requirements are greater than those of normalweight infants ${ }^{2}$. Randomised clinical trials have shown that these greater nutritional needs may persist beyond the neonatal period ${ }^{3,4}$. However, evidence-based research on the association between early milk and solid feeding in LBW infants and subsequent development and health during childhood and adulthood is limited. Indeed, the

†Current address: Medical Research Council Environmental and Epidemiological Unit, University of Southampton, Tremona Road, Southampton SO16 6YD, UK. accepted wisdom that the promotion of preterm infant growth is ideal for optimal health and development has been reviewed and challenged ${ }^{5}$. This uncertainty about 'optimal' nutritional needs may lead health-care professionals and others to give conflicting messages to those responsible for the feeding and care of LBW infants.

The 2000 Infant Feeding Survey ${ }^{6}$ showed that infant feeding practices are commonly disparate from those that would be expected if official guidance, e.g. the Department of Health's Weaning and the Weaning Diet ${ }^{7}$, were followed. Furthermore, a study of full-term infants in 1990 showed important misconceptions regarding desirable feeding practice amongst mothers ${ }^{8}$. While much is known about overall infant feeding practices in the UK, there is 
very little recorded about practices among carers of LBW infants. Information concerning feeding patterns of LBW infants is important from a public health perspective because any divergence from customary nutritional practice may have more profound long-term significance for LBW than for normal-birth-weight infants, in terms of growth $^{9}$ and later cognitive development ${ }^{10}$. In addition, this information could help in the design of further randomised controlled trials (RCTs) to explore the optimum nutritional strategy for LBW infants after hospital discharge. In fact, as a result of the findings of the present survey, the authors initiated an RCT which explored the effects of a novel feeding regimen on growth and health outcomes ${ }^{4}$.

The aims of the present work were to describe feeding practices used in LBW infants after hospital discharge along with mothers' perceptions of desirable feeding practices, followed by testing null hypotheses of no association between the favouring of inappropriate feeding regimens and demographic features, in order to identify a potential 'high-risk group' of mothers to whom optimal LBW feeding information should be especially targeted.

\section{Subjects and methods}

\section{Subjects}

Between 1997 and 1998 the mothers of LBW infants (defined as having a recorded birth weight of $2500 \mathrm{~g}$ or less) born at three hospitals in England (Royal Hampshire County Hospital in Winchester, Liverpool Women's Hospital and Chelsea \& Westminster Hospital in London) were contacted by post or directly by a designated member of the nursing staff and the study aims were explained. If the mother expressed an interest in the study, a copy of the questionnaire together with a covering letter and a pre-paid envelope for return of the questionnaire were sent to her home address. The questionnaire, when completed, was returned to the principal investigator (L.D.M.) at the University of Surrey.

In order to achieve a satisfactory basis for statistical analysis, using a scenario of performing a one-sample twotailed test for a standardised difference of 20\% with significance of $5 \%$ and power of $80 \%$, it was deemed that at least 197 completed questionnaires would be required (AC Kimber, Statistician, personal communication).

\section{The questionnaire}

Closed questions on the types of milk used and the timing of the introduction of different solid foods for the LBW infants realised the two feeding practice outcomes below:

- Whether or not the mother had ever breast-fed her infant;

- Age of the infant when solid food was first introduced.
Closed questions on healthy infant feeding regimes contained seven key questions, i.e. seven attitudes outcomes identifying the correctness of mothers' perceptions of good infant diet in relation to:

- Wide variety of foods;

- Low sugar intake;

- Additive-free foods;

- Low salt intake;

- High fibre intake;

- Low fat intake;

- 'Plenty of calories'.

Closed and open demographic questions included the following five potential predictors for these outcomes:

- Mother's age;

- Social group of the family based on the principal wage earner's current or previous occupation ${ }^{11}$;

- Mother's parity (first or subsequent birth);

- Infant's gender;

- Infant's birth weight.

Other potential demographic predictors were eliminated owing to strong correlation with one of the above (e.g. infant's gestational age at birth: highly correlated (Pearson's correlation test: $r=+0.858, P<0.001$ ) with infant birth weight), poor compliance in responding to the relevant question (e.g. household income bracket: 34 (17.2\%) missing responses) or a scarcity of subjects in one category of a dichotomous variable (e.g. only 15 (7.7\%) mothers followed a vegetarian or vegan diet).

The questionnaire was based on one designed and successfully administered for mothers of term infants ${ }^{8}$ and was amended after a pilot study in which mothers from a National Childbirth Trust group in Hampshire completed the questionnaire and submitted comments to L.D.M.

\section{Etbical approval}

Approval to conduct the study was obtained from the local research ethics committees of each of the three participating hospitals.

\section{Statistical analysis}

Data from the closed questions were entered and verified by the principal investigator (L.D.M.). Open questions were collated manually. Summary statistics for demographics are reported along with milk feeding practices, solid food introduction and mothers' attitudes to appropriate infant feeding practices. The derived percentages are based upon the total number of responses to each question; not all questions were completed by all mothers. Each of the nine outcome variables specified above was analysed for its association with the five specified demographic variables. Appropriate statistical tests were employed in accordance with the nature of the variables under analysis and are described fully in the Results 
section. SPSS (version 10; SPSS Inc., Chicago, IL, USA) was used for statistical analysis.

\section{Results}

Of the recruited 364 mothers of LBW infants, 198 (54.4\%) returned completed questionnaires.

\section{Subjects}

The mothers' median age at the time of completing the questionnaire was 31 years (range 16-45 years). Based on the mothers' occupations, 79 (39.9\%) were classified as social group I or II, $72(36.3 \%)$ as IIINM or IIIM, and 17 (8.6\%) as IV or V. The remaining 30 (15.1\%) failed to list their job title, or recorded 'student' or 'unemployed'. The majority of mothers lived with a partner/husband (169, $86.7 \%)$, were of white ethnic origin $(161,89.0 \%)$ and had had a singleton birth (158, 81.9\%); 113 (61.7\%) reported that they were first-time mothers.

One hundred and twelve (56.6\%) of the infant population were girls. The infants' median age was 10 months (range 5-17 months). At birth, the infants' median gestational age was 33 weeks (range 24-42 weeks) and median weight was $1.80 \mathrm{~kg}$ (range $0.6-2.5 \mathrm{~kg}$ ); $2.5 \%$ were born in 1996, 81.3\% in 1997 and 16.2\% in 1998.

\section{Milk feeding practices}

Trends in milk feeding practices are displayed in Fig. 1. Seventy-two mothers (37.1\%) reported exclusive breast-feeding (no other food or drinks) of their infant from birth to 4 weeks; by 6 months of age, $16.0 \%$ reported

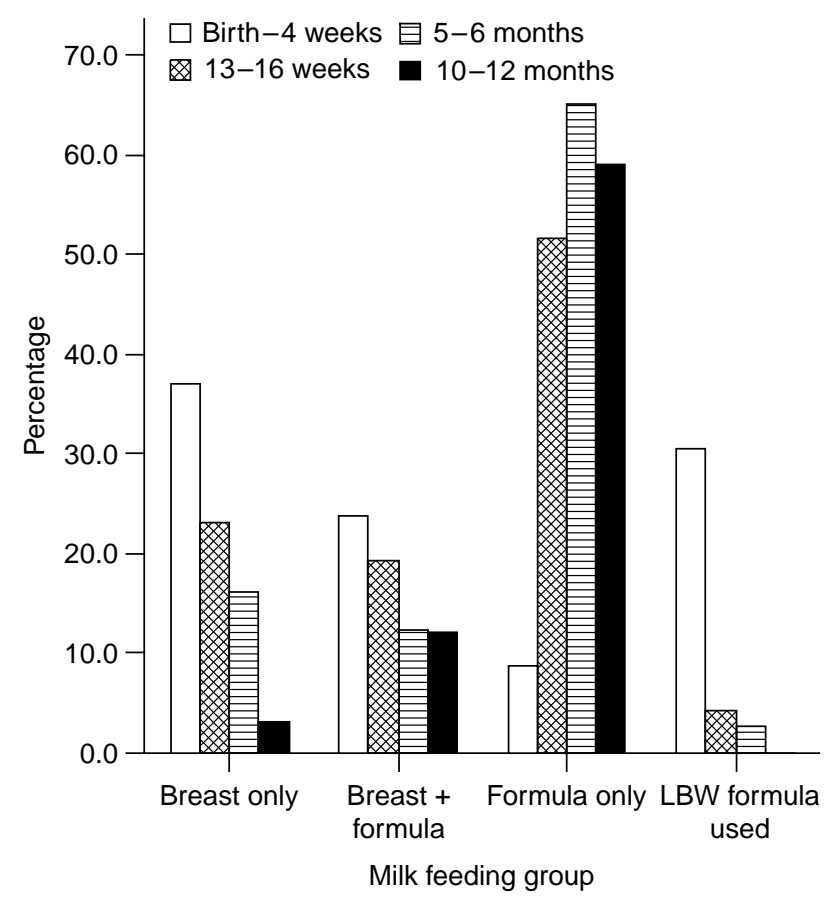

Fig. 1 Milk feeding patterns (breast, infant milk formula and lowbirth-weight (LBW) infant formula) over the first 12 months in LBW infants using breast milk as the only milk fed to their infants, although all infants by this time were receiving other foods or drinks. The proportion of infants fed a combination of breast and standard infant milk formula decreased from $23.7 \%(n=46)$ between birth and 4 weeks to $12.3 \%$ at 6 months. The proportion of infants fed only standard infant milk formula increased from $8.8 \%(n=17)$ between birth and 4 weeks to $65.2 \%$ at 6 months. The proportion of infants fed an LBW formula (alone or in tandem with breast-feeding) decreased from $30.4 \%(n=59)$ between birth and 4 weeks to $2.7 \%$ at 6 months; by this time the prevalence of any breast-feeding amongst LBW formula recipients had declined from $57.6 \%$ to $20.0 \%$.

Of the 196 mothers who provided complete milk feeding histories, $164(83.7 \%)$ breast-fed at some stage and 32 (16.3\%) did not. The associations of ever breast-feeding with the five potential demographic predictors are summarised in the relevant column of Table 1. Statistically significant associations were found for two demographic predictors:

- Social group - lower social group was associated with not having ever breast-fed [never breast-fed ( $n=24$, median social group $=$ IIINM, range I to V) vs. breastfed $(n=142$, median social group $=$ II/IIINM, range I to V), Mann-Whitney $U$-test: $P=0.019$ ];

- Mother's parity - of 112 mothers of first infants, 101 (90.2\%) breast-fed compared with 51 of 69 (73.9\%) multiparous mothers (chi-square test: $P=0.004$ ).

\section{Solid feeding practices}

The median age at solid food introduction was 17 postnatal weeks (range 8-36 weeks) and the median gestational-corrected age was 11 weeks (range -1 to 27 weeks). The median weight of the infants when solids were introduced was $5.00 \mathrm{~kg}$ (range $2.49-7.48 \mathrm{~kg}$ ); $50 \%$ received solid foods before they achieved $5 \mathrm{~kg}$. By 16 postnatal weeks, 89 (47.6\%) mothers had introduced solid foods. Baby rice was the most frequently used first weaning food, either alone (82 infants, 42.9\%) or in combination with another food (75 infants, 39.3\%). For 19 (9.9\%) additional infants, combinations of other foods (not including baby rice) were used.

The associations of infant age at solid food introduction with the five potential demographic predictors are summarised in the relevant column of Table 1. A statistically significant association was found only for one demographic predictor:

- Infant birth weight - a statistically significant negative correlation with infant age at solid food introduction was found (Pearson's correlation test: $r=-0.293, P<0.001$ ).

\section{Attitudes of mothers to appropriate infant feeding practices}

Mothers were asked to gauge the importance of certain dietary practices for infants on a scale of 'very important', 
'important' or 'not important' (Table 2, 1997-1998 columns). One hundred and eighty-two (91.9\%) mothers rated a 'wide variety of foods' as important or very important. The figures for rating as important (or very important) a low sugar intake, a low salt intake, high fibre intake and a low fat intake were 176 (88.8\%), 182 (91.9\%) $133(67.1 \%)$ and 102 (51.6\%), respectively. Forty-nine (24.7\%) mothers considered that 'plenty of calories' was not important; upon comparison within individual responses, 79 (39.9\%) assigned greater importance to an additive-free diet than to 'plenty of calories'.

The five potential demographic predictors were analysed for associations with the seven feeding regimens. The results are summarised in the seven right-hand columns of Table 1 . No statistically significant associations were found except for the following pairings:

- Social group and low fat intake - lower social groups were associated with the (incorrect) assignment of a higher level of importance to low fat intake (Spearman's rank correlation test: $P=0.018$ );

- Mother's parity and high fibre intake - multiparous mothers were more likely to assign (incorrectly) a high level of importance to high fibre intake than first-time mothers (Mann-Whitney $U$-test: $P=0.006$ );

- Mother's parity and 'plenty of calories' - first-time mothers were more likely to assign (incorrectly) a low level of importance to 'plenty of calories' than multiparous mothers (Mann-Whitney $U$-test: $P=0.047$ );

- Infant birth weight and 'plenty of calories' - higher infant birth weight was associated with the (incorrect) assignment of a lower level of importance to 'plenty of calories' (Spearman's rank correlation test: $P=0.004$ ).

\section{Discussion}

\section{Long-term effects of early nutrition}

In all LBW infants appropriate nutrition is important in the early days of life for adequate brain and somatic growth.

Table 2 Mothers' attitudes to infant feeding practices in the current survey of low-birth-weight infants $(1997-1998 ; n=198)$ compared with a survey of term infants $(1990 ; n=1000)$

\begin{tabular}{lcccccc}
\hline & \multicolumn{2}{c}{ Not important } & & \multicolumn{2}{c}{$\begin{array}{c}\text { Important/very } \\
\text { important }\end{array}$} \\
\cline { 2 - 3 } \cline { 5 - 6 } \cline { 5 - 6 } & 1990 & $1997-1998$ & & 1990 & $1997-1998$ \\
\hline Wide variety of foods & 3.2 & 2.0 & & 95.3 & 91.9 \\
Low sugar intake & 2.0 & 7.1 & & 96.9 & 88.8 \\
Additive-free & 5.5 & 9.6 & & 92.3 & 84.4 \\
Low salt intake & 3.1 & 4.0 & & 95.8 & 91.9 \\
High fibre & 14.4 & $26.8^{*}$ & & 83.2 & 67.1 \\
Low fat intake & 10.2 & $41.4^{\star *}$ & & 87.5 & 51.6 \\
'Plenty of calories' & 20.2 & 24.7 & & 76.4 & 70.2 \\
\hline
\end{tabular}

Results are given as a percentage of mothers' overall responses. Data on non-responders not provided.

* Greater proportion of mothers in 1997-1998 than in 1990 correctly identified the inappropriateness of a high-fibre diet (chi-square test: $P<0.001$ ).

** Greater proportion of mothers in 1997-1998 than in 1990 correctly ident-

ified the inappropriateness of a low-fat diet (chi-square test: $P<0.001$ ). 
However, paradoxically, relative undernutrition (in preterm infants) may confer an advantage in some of the physiological markers which in the long term promote cardiovascular health ${ }^{5}$. Thus, knowledge of the determinants of feeding practices of LBW infants and evidencebased guidelines for the feeding of LBW infants are essential, both to promote growth and development in infancy and childhood and for the potential significance of early feeding on long-term health outcomes such as blood pressure, dyslipidaemia, obesity, diabetes and metabolic syndrome.

In the present study we investigated mothers' attitudes to infant feeding practices. It was not anticipated that our sample would be representative of mothers living in England and Wales, given the self-selecting nature of the study that involved completing a questionnaire relating to infant feeding practices and knowledge of healthy eating for infants. Not surprisingly there was a wide range of practice. As a result of this survey, we subsequently designed and executed an RCT to develop guidelines for solid feeding introduction in preterm infants to optimise growth and development ${ }^{4}$. These guidelines have yet to be adopted on a national basis although they have been implemented in one Health Trust and have been incorporated into 'Weaning your Premature Baby' by the premature baby charity, BLISS.

\section{Subjects}

In our survey the sample consisted of 198 mother/LBW infant pairs and was recruited from three different locations in England. The response rate, 54.4\%, was in line with rates reported by us in questionnaires on mothers living in South East England undertaken at around the same time ${ }^{12}$. As there was insufficient information on the proportions of infants who were preterm, term or small-for-gestationalage within the LBW population, we were unable to undertake separate statistical analysis on these subgroups.

The social make-up of the sample was based on the mothers' current occupation using the 1991 classification of the Office of Population Censuses and Surveys ${ }^{11}$ and was broadly in line with the social make-up of the 1990 survey $^{8}$, where the socio-economic groupings were described using the same classification. This enabled us to make comparisons of the 'attitudes' data from the two surveys (see below). The mothers' age range (16-45 years) in the present study was similar to that (16-44 years) of the 1990 study $^{8}$.

\section{Milk feeding practices}

The rate of provision of breast milk initially, to $83.7 \%$ of the infants in this study, was considerably higher than the national rates of $69 \%$ for term infants, $71 \%$ for LBW infants and $68 \%$ for infants admitted to neonatal units reported in the 2000 survey $^{6}$. Even so, no infant in the present study fulfilled the subsequently introduced recommendation that breast milk should provide the sole source of nutrients to 6 months of age ${ }^{13}$. Not unexpectedly, and in line with factors associated with breast-feeding of term infants, occupations of higher social classification were associated with initial and continued breast-feeding. The finding that mothers of first infants were more likely to attempt to breast-feed their LBW infant than multiparous mothers reflects findings from the 2000 UK national survey. Here, mothers of first infants who were full term were reported to be more likely to initiate breast-feeding than others, although by 6 weeks this trend had begun 'to even out' ${ }^{6}$. In summary, mothers from the lower social groups and multiparous mothers need to be targeted regarding the benefits of breast-feeding.

\section{Solid feeding practices}

The median age at which weaning began was 17 postnatal weeks (range 8-36 postnatal weeks) and the median gestational-corrected age was 11 weeks (range -1 to 27 weeks). Thus, 50\% of mothers were not following the recommendation in force at the time of the survey ${ }^{7}$ for breast-fed and formula-fed term infants, which was to introduce solids between 4 months (17 weeks) and 6 months (26 weeks) for the majority of infants ${ }^{7}$. In addition, there was a wide diversity in practice of solid food introduction. These findings are in broad agreement with those of Norris et al. ${ }^{14}$, who studied 257 preterm infants from South East England between 1996 and 1998, where weaning age ranged from 5 to 36 weeks after birth. Our results also reflect those of Fewtrell et al..$^{15}$ from a cohort of 492 preterm infants recruited in the mid-1990s where weaning age ranged from 10 to 36 postnatal weeks. In the national 2000 survey of 362 LBW infants ${ }^{6}, 32 \%$ of infants had received solid foods by 16 weeks, a lower proportion than that observed in the present study (48\%).

Our results for the infant weight achieved at first introduction of solid foods also indicates poor compliance (50\%) with the specific contemporary recommendations by the Department of Health ${ }^{7}$ for LBW infants, which is 'when an infant weighs at least $5 \mathrm{~kg}$ '. In a similar study of preterm infants, $21 \%$ were weaned before achieving a body weight of $5 \mathrm{~kg}^{14}$. In addition, food diversity was commonly practised by mothers in our sample, with 39\% combining foods as first weaning foods. However, the practice of early food diversity has since been found to be associated with eczema development at 12 months in some preterm infants ${ }^{16}$.

The range of practice with respect to solid feeding may not be surprising for several reasons. LBW infants have high energy and nutrient requirements, which may not be satisfied by milk feeding alone for 6 months from birth. Second, national ${ }^{7}$ and international ${ }^{13}$ recommendations may not be followed because they are not evidencebased $^{17}$. In fact, the relevance of the World Health Organization code on exclusivity of breast-feeding to 6 months to all infants, the 'one size fits all approach', must be questioned for LBW infants reared in industrialised 
countries $^{18}$. These families may not be receiving sufficient advice or support on the best feeding regimen for their infant. In summary, the infant's size and development appear to dominate over the mother's social and demographic background in her decision as to when to start solid feeding, as indicated by the strong correlation between infant birth weight and subsequent time at which solid food was introduced.

\section{Attitudes of mothers to appropriate infant feeding practices}

Several 'high-risk' demographic groups were identified in relation to incorrect perceptions of good infant feeding regimens. Mothers of lower social groups overestimated the importance of a low-fat diet, multiparous mothers overestimated the importance of high fibre intake, while first-time mothers underestimated the importance of plenty of calories, as did mothers of heavier infants.

The responses to the mothers' attitudes questions were compared (Table 2) with those obtained from a 1990 survey of 1000 mothers of term infants living in England ${ }^{8}$. The proportion of mothers who correctly interpreted good infant feeding practices, such as importance of a wide variety of foods and low sugar, low salt intake, was similar in both surveys. More mothers in this LBW survey than in the 1990 survey correctly identified that a low-fat (chisquare test: $P<0.001$ ) and high-fibre diet (chi-square test: $P<0.001$ ) was inappropriate. However, a quarter of mothers in our survey responded incorrectly that 'plenty of calories' was not important, representing a decrease, albeit not statistically significant, in the correct response compared with 20\% in the 1990 cohort (chi-square test: $P=0.151$ ).

Inappropriate maternal beliefs of infant feeding principles may affect the nutrition of any infant but are particularly worrying if held by the mothers of LBW and preterm infants, such as those in our survey. Many of these infants will have growth deficits which may need to be mitigated by the provision of high-energy/nutrient-dense diets. This snapshot of mothers' attitudes shows that the interpretation of 'healthy eating' for infants by mothers is frequently incorrect. Sadly this state of affairs has not improved greatly from the early 1990s. However, here we have been able to identify specific subgroups of mothers who are prone to certain inappropriate practices and misconceptions in relation to the health of LBW infants. In particular, multiparous mothers appear to forsake breastfeeding for infant formula feeding (maybe with legitimate although unspecified cause) and also adopt an attitude to a 'healthy' infant diet (specifically in the form of high fibre intake) which is more appropriate for older children, possibly influenced/confused by the concomitant needs of their older children. In summary, this is a complex picture where social group and demographic background have an influence on the understanding of good infant feeding practices.

\section{Conclusions}

We found a wide range of infant feeding practices, varying levels of knowledge and differing attitudes about infant feeding amongst mothers of LBW infants post-discharge from the neonatal unit. Existing yardsticks for good infant feeding practice for LBW infants should be used to formulate clear guidelines for use among health professionals advising the carers of LBW infants in industrialised countries. In particular, mothers of lower social groups need to be targeted with regard to the benefits of breast-feeding, in particular initially, as well as with regard to the lack of necessity for low fat intake in LBW infants. Furthermore, all multiparous mothers need to be reminded of the benefits of breast-feeding and also of the lack of necessity for high fibre intake in their LBW infants. Finally, the benefit of 'plenty of calories' also needs to be affirmed.

\section{Acknowledgements}

We would like to express our gratitude to the families for their participation in this study and to the neonatal midwives, nurses, dietitians and administrators who set up systems to enable the survey families to be approached, or who approached families on our behalf. Our particular thanks go to Cathy Williams and Lilian Pekarik (Royal Hampshire County Hospital), Jerry McGee and Tracey Reeves (Chelsea \& Westminster Hospital) and Jenny Rogahn (Liverpool Women's Hospital).

We also wish to extend thanks to Dr Alan Kimber for providing the initial statistical support and Professor JWT Dickerson for invaluable comments on the script. We acknowledge Cow \& Gate Nutricia with grateful thanks for PhD sponsorship for L.D.M., and in particular the advice received from John Wells.

A copy of the questionnaire can be obtained on request from the corresponding author.

\section{References}

1 Barker DJ. Early growth and cardiovascular disease. Archives of Disease in Childhood 1999; 80: 305-10.

2 Tsang RC, Lucas A, Uauy R, Zlotkin S, eds. Consensus recommendations. In: Nutritional Needs of the Preterm Infant. Baltimore, MD: Williams \& Wilkins, 1993; 288-95.

3 Lucas A, Fewtrell MS, Morley R, Singhal A, Abbott RA, Isaacs $\mathrm{E}$, et al. Randomized trial of nutrient-enriched formula versus standard formula for postdischarge preterm infants. Pediatrics 2001; 108: 708-11.

4 Marriott LD, Foote KD, Bishop JA, Kimber AC, Morgan JB. Weaning preterm infants: a randomised controlled trial. Archives of Disease in Childhood. Fetal and Neonatal Edition 2003; 88: F302-7.

5 Singhal A, Lucas A. Early origins of cardiovascular disease: is there a unifying hypothesis? Lancet 2004; 363 : $1642-5$ 
6 Hamlyn B, Brooker S, Lleinikova K, Wands S. Infant Feeding 2000. London: TSO, 2000.

7 Department of Health. Weaning and the Weaning Diet. Report on Health and Social Subjects No. 46. London: HMSO, 1994.

8 Morgan JB, Kimber AC, Redfern AM, Stordy BJ. Healthy eating for infants - mothers' attitudes. Acta Paediatrica 1995; 84: 512-5.

9 Lucas A, Morley R, Cole TJ, Gore SM, Lucas PJ, Crowle P, et al. Early diet in preterm babies and developmental status at 18 months. Lancet 1990; 335: 1477-81.

10 Lucas A, Morley R, Cole TJ. Randomised trial of early diet in preterm babies and later intelligence quotient. British Medical Journal 1998; 317: 1481-7.

11 Office of Population Censuses and Surveys (OPCS). Standard Occupational Classification, Vol. 3. Social Classifications and Coding Methodology. London: OPCS, 1991.

12 Rasasi B-Al. Nausea and vomiting in pregnancy: maternal nutrition and pregnancy outcome. PhD thesis, University of Surrey, Guildford, UK, 2003.
13 World Health Organization (WHO). Infant and young child nutrition. Resolution 54.2, Fifty-fourth World Health Assembly. Geneva: WHO, 2001.

14 Norris FJ, Williams CM, Larkin M, Hampton SM, Morgan JB. Factors affecting the introduction of complementary feeding in the preterm infant. European Journal of Clinical Nutrition 2002; 56: 448-54.

15 Fewtrell MS, Lucas A, Morgan JB. Factors associated with weaning in full term and preterm infants. Archives of Disease in Childhood. Fetal and Neonatal Edition 2003; 88: F296-F301.

16 Morgan J, Williams P, Norris F, Williams CM, Larkin M, Hampton S. Eczema and early solid feeding in preterm infants. Archives of Disease in Childhood 2004; 89: 309-14.

17 Lanigan JA, Bishop J, Kimber AC, Morgan J. Systematic review concerning the age of introduction of complementary food to the health full-term infant. European Journal of Clinical Nutrition 2001; 55: 309-20.

18 Foote K, Marriott LD. Weaning of infants. Archives of Disease in Childhood 2003; 88: 488-92. 